\title{
Allosteric Inhibition of Adenylyl Cyclase Type 5 by G-Protein: A Molecular Dynamics Study
}

\author{
Elisa Frezza ${ }^{1, *(\mathbb{D}}$, Tina-Méryl Amans ${ }^{2}\left(\mathbb{D}\right.$ ) and Juliette Martin ${ }^{2, *(\mathbb{D}}$ \\ 1 CiTCoM, CNRS, Université de Paris, F-75006 Paris, France \\ 2 CNRS, UMR 5086 Molecular Microbiology and Structural Biochemistry, University of Lyon, F-69367 Lyon, \\ France; amans.tina@gmail.com \\ * Correspondence: elisa.frezza@u-paris.fr (E.F.); juliette.martin@ibcp.fr (J.M.); \\ Tel.: +33-1-53-73-15-69 (E.F.); +33-4-37-65-29-45 (J.M.)
}

Received: 10 July 2020; Accepted: 16 September 2020; Published: 17 September 2020

\begin{abstract}
Adenylyl cyclases (ACs) have a crucial role in many signal transduction pathways, in particular in the intricate control of cyclic AMP (cAMP) generation from adenosine triphosphate (ATP). Using homology models developed from existing structural data and docking experiments, we have carried out all-atom, microsecond-scale molecular dynamics simulations on the AC5 isoform of adenylyl cyclase bound to the inhibitory G-protein subunit G $\alpha \mathrm{i}$ in the presence and in the absence of ATP. The results show that G $\alpha$ i has significant effects on the structure and flexibility of adenylyl cyclase, as observed earlier for the binding of ATP and Gs $\alpha$. New data on G $\alpha$ i bound to the C1 domain of AC5 help explain how Gai inhibits enzyme activity and obtain insight on its regulation. Simulations also suggest a crucial role of ATP in the regulation of the stimulation and inhibition of AC5.
\end{abstract}

Keywords: molecular dynamics; flexibility; protein-protein interactions; allostery; enzyme activity; docking; homology models

\section{Introduction}

One of the most studied signal transduction pathways is the intricate control of cyclic AMP (cAMP) generation, which is a universal second messenger based on G-protein coupled receptors (GPCR) in eukaryotes [1]. cAMP has a role in a vast number of biological systems, including but not limited to hormone secretion [2], smooth muscle relaxation [3], olfaction [4], learning, and memory [5-7].

The family of enzymes responsible for cAMP synthesis are the adenylyl cyclases (also commonly known as adenylate cyclases), which are highly regulated in order to tightly control cAMP levels [8]. Nine mammalian transmembrane ACs are recognized (hereafter termed AC1-9), with a cytoplasmic domain with catalytic properties [8]. Each member of the family has specific regulatory properties and tissue distributions $[9,10]$; however, they all convert adenosine triphosphate (ATP) into cAMP via a cyclization reaction.

Mammalian ACs share a similar topology of a variable N-terminus (NT) and two repeats of a membrane-spanning domain followed by a cytoplasmic domain [11,12]. The two cytoplasmic domains, called $\mathrm{C} 1$ and $\mathrm{C} 2$, contain a region of approximately 230 amino acid residues that are roughly $40 \%$ identical, which are called C1a and C2a: This implies a pseudosymmetry in ACs. Together, the cytoplasmic domains form the catalytic moiety at the interface. The NT and C-terminal portion of the $\mathrm{C} 1$ and $\mathrm{C} 2$ domains, called $\mathrm{C} 1 \mathrm{~b}$ and $\mathrm{C} 2 \mathrm{~b}$, are the most variable regions among the different isoforms and can differ among the species. The catalytic site of ACs is located at the C1/C2 interface and binds a molecule of ATP accompanied by two magnesium ions [13]. 
ACs' function is regulated by several modulators, which are either stimulators or inhibitors of cAMP synthesis. These include the stimulatory G-protein subunit alpha (Gs $\alpha)$, which is released from its cognate receptor and binds to and activates the AC enzyme via the subunit interaction with the C2 domain [10,14-16] upon GPCR activation [14,16,17], the inhibitory G-protein subunits Gai and $\mathrm{G} \beta \gamma$, calcium ions, calmodulin, and a variety of kinases. AC isoforms integrate several signals and they differ from each other for their modulators and for the different tissues where they are more abundant [18-21]. Although all nine transmembrane ACs are expressed in the brain, specific ACs are particularly abundant in specific brain regions, and AC5 is highly expressed in the striatum; therefore, it seems to be involved in signal transduction networks that are crucial for synaptic plasticity in the two types of medium spiny neurons [22].

Structural information on the AC cytoplasmic catalytic core [21] and on a complex containing both AC catalytic domains bound to an active conformation of the stimulating Gs $\alpha$, with or without a bound ATP analog, is available [23]. However, the transmembrane regions contain six predicted membrane-spanning helices each and their function, aside from membrane localization, is unknown. Although the mechanism of stimulation of AC by Gs $\alpha$ is relatively well understood, the mechanism of inhibition of AC activity is still debated: some mutational studies suggest that G $\alpha$ i binds in an opposite binding site [24], but there are other hypotheses, such as the possibility of the simultaneous binding of $\mathrm{G} \alpha \mathrm{i}$ and $\mathrm{Gs} \alpha$ or a competition between the two G proteins. However, there are no data on the enzyme bound to ATP (or an ATP analog) in the absence of activating Gs $\alpha$, on the enzyme in complex with G $\alpha \mathbf{i}$ in the presence or absence of ATP and on the possible trimeric form G $\alpha \mathrm{i}+\mathrm{AC}+\mathrm{Gs} \alpha$ in the presence or absence of ATP. Hence, it is difficult to understand how G $\alpha$ subunits activate/inhibit adenylyl cyclase and what is the role of ATP.

To gain insight into the functional mechanism of AC, some studies at the molecular level have been conducted using all-atom molecular dynamics (MD) simulations. In our previous work, we studied the stimulation mechanism of AC5 by performing MD simulations of AC5 alone, AC5 + ATP, AC5 + Gs $\alpha$, and AC5 + ATP + Gs $\alpha$ [25]. We chose the mouse AC5 isoform among the other isoforms, since this isoform notably plays a key role in a variety of neuronal GPCRs-based signal cascades [19,26,27]. We extensively characterized the flexibility of the four states, the protein-protein interfaces, the ATP mobility, the Gs $\alpha$ binding site, and the G $\alpha$ i putative binding site on C1 and the effect of ATP and Gs $\alpha$ on these properties. Our study showed that both ATP and Gs $\alpha$ binding have significant effects on the structure and flexibility of adenylyl cyclase. The comparison between the simulations of AC5 + ATP and AC5 + ATP + Gs $\alpha$ helped to explain how Gs $\alpha$ binding enhances enzyme activity and could therefore aid product release. Our simulations also suggested that ATP binding could influence the binding of the inhibitory G-protein subunit G $\alpha \mathrm{i}$, if the potential binding site within domain $\mathrm{C} 1$ were to be involved.

At the same time, another study by Van Keulen and co-workers has been published where they investigated the mechanism of inhibition of AC5 by N-terminal myristoylated G $\alpha$ i. In their studies, they considered apo AC5 (i.e., without ATP), and they concluded that the myristoylation seems to play

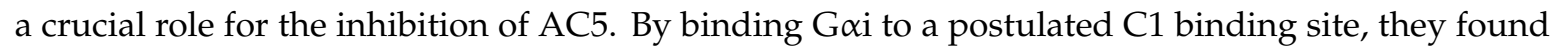
structural modifications that would disfavor both ATP and Gs $\alpha$ binding [28]. Recently, they have also characterized the complex G $\alpha \mathrm{i}+\mathrm{AC} 5+\mathrm{Gs} \alpha$ using N-terminal myristoylated G $\alpha \mathrm{i}[29,30]$ by comparing the different simulations in order to understand the impact of the binding of both $G \alpha$ proteins. This comparison suggests that the association of both $\mathrm{G} \alpha \mathrm{i}$ and $\mathrm{Gs} \alpha$ subunits results in an AC5 conformation similar to that sampled by the G $\alpha \mathrm{i}+\mathrm{AC} 5$ complex, indicating that the ternary complex mainly samples an inactive conformation.

Despite these recent studies, the impact that Goi would have if ATP were already bound in its AC5 pocket and also whether Gs $\alpha$ and G $\alpha$ i could nevertheless bind simultaneously to AC5 in the presence of ATP are yet to be clarified. In the present study, we used the same approach applied to investigate the stimulation mechanism in our previous work [25]. We have used all-atom molecular dynamics simulations to study the impact of ATP and Gai on the structure and flexibility of AC5. As in 
our previous study, we considered only the cytoplasmic domains of AC5, since they are capable of reproducing many of the regulatory properties of the wild-type enzyme and therefore can be used as working models to investigate the regulation mechanisms of AC [31,32]. Since no structural data are available for the complex AC5 + G $\alpha$ i, we computed docking experiments using representative structures for G $\alpha$ i and AC5 + ATP obtained from our MD simulations, and we considered two distinctive poses. The all-atom microsecond-scale simulations of AC5 in complex with G $\alpha$ i with or without ATP studied here (see Figure 1) were compared with our previous simulations of AC5, $\mathrm{AC} 5+\mathrm{ATP}, \mathrm{AC} 5+\mathrm{Gs} \alpha$, and AC5 + ATP + Gs $\alpha$ in order to help explain how binding changes the

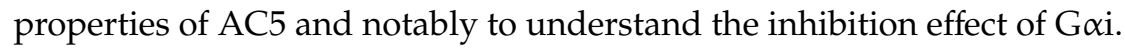

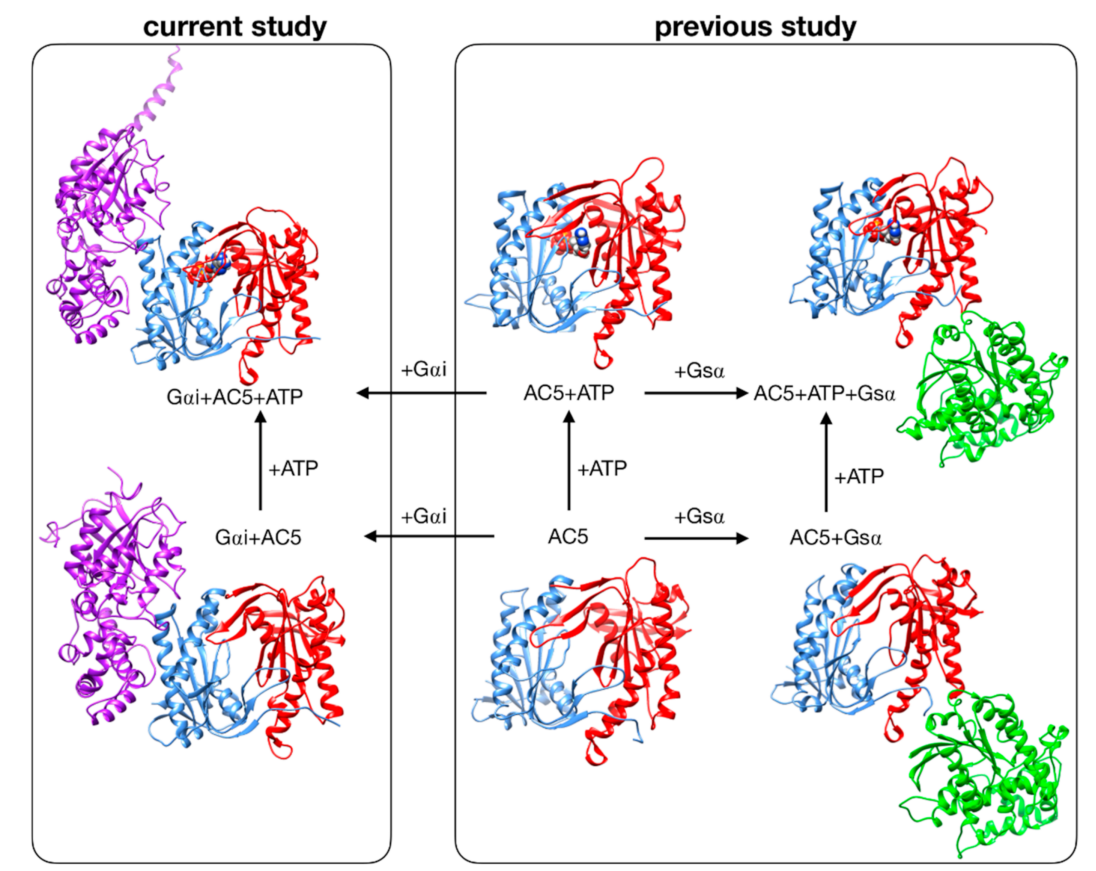

Figure 1. Structure of the cytoplasmic segment of the AC5 isoform of adenylyl cyclase and of its complexes with ATP and the regulating G-proteins Gs $\alpha$ and G $\alpha$ i viewed from the side closest to the cell membrane. Proteins are shown as backbone ribbons. The $\mathrm{C} 1$ and $\mathrm{C} 2$ subunits of $\mathrm{AC} 5$ are colored blue and red respectively, Gs $\alpha$ is colored green, and G $\alpha \mathrm{i}$ is colored purple. ATP is shown in a CPK representation with standard chemical coloring. In each case, the structures are averages taken from the molecular dynamics simulations. For the AC5 in complex with G $\alpha$ i with and without ATP, we chose one of the docking poses we used in this work.

\section{Materials and Methods}

\subsection{Models}

Models of the cytoplasmic domains of AC5 and Gai protein were built by homology to known proteins using Modeller v9.12 [33]. In each case, 100 homology models were generated, and the model with the lowest DOPE score [34] was selected.

For AC5, the model was taken from the MD simulation of our previous study [25] by considering the structure closest to the center of the largest cluster of the last 500 ns simulation of AC5 + ATP. The model we studied earlier [25] was a homology model for the cytoplasmic domains of mouse AC5 (UniprotKB-P84309) with bound ATP, which was generated using the structure 1CJK [13] as template (template/model sequence identity was 98\% for the C1 domain, spanning residues 455-643 of P84309, and 57\% for the C2 domain, spanning residues 1063-1257 of P84309).

For G $\alpha \mathrm{i}$, a homology model spanning residues 9-355 of the mouse sequence (UniprotKB-P08752) bound with GSP (a GTP analogue) and $\mathrm{Mg}^{2+}$ ion was generated using Modeller with three templates: 
1CJK [13] (bovine Gs $\alpha 38 \%$ sequence identity), 1AS3 [35] (rat G $\alpha \mathrm{i}, 81 \%$ identity), and 1AGR [36] (rat G $\alpha$ i, $87 \%$ sequence identity). This model was used in docking (see below). In addition, we considered a model of G $\alpha$ i sampled from MD simulation: a simulation of $1 \mu$ s was run starting from the homology model. The structure closest to the center of the largest cluster observed during the simulation was used for docking. In physiological condition, G $\alpha$ i has post-translational modifications, including a myristoylation on its N-terminus tail that is required for the inhibition of AC5, which affects the

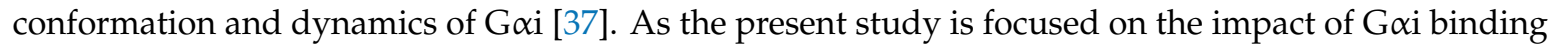
on the dynamics and conformation of AC5, we did not include such myristoylation in our model of G $\alpha$ i.

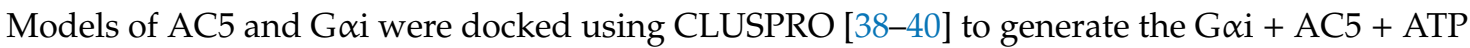
complexes shown in Figure 2, by setting as "attractive residues" (see Figure S1) some residues in the proximity of the presumed binding site from ref [24]. These residues are subject to extra attractive forces during the docking calculation [40].
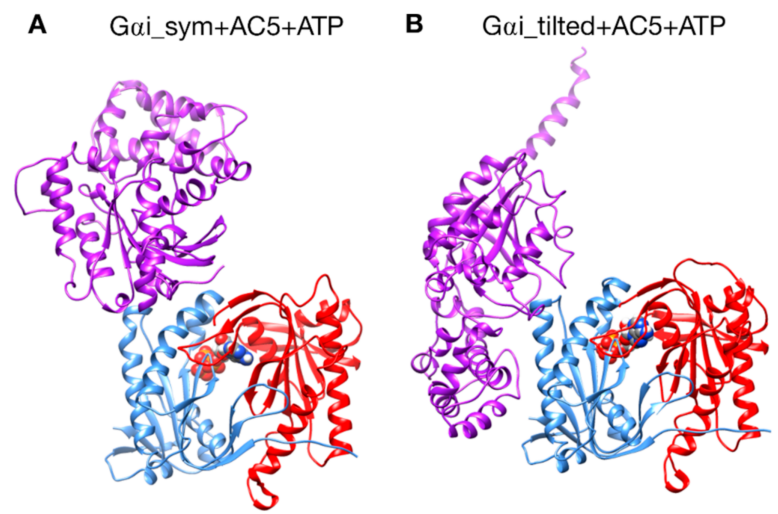

Figure 2. The two different configurations of the G $\alpha \mathrm{i}+\mathrm{AC} 5+\mathrm{ATP}$ complex simulated in this study. (A): G $\alpha$ i_sym + AC5 + ATP: G $\alpha$ i has an orientation symmetrical to the Gs $\alpha$ protein in the AC5 + Gs $\alpha$

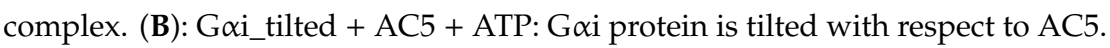

A first complex was built by docking the model of AC5 sampled from simulation with the

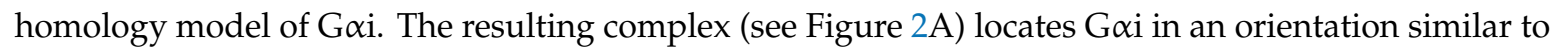
Gs $\alpha$ with respect to AC5 in the 1CJK complex, with the G $\alpha$ i protein binding in the groove formed by the two $\alpha$-helices termed $\alpha 1$ and $\alpha 2$ in Figure 3. This orientation is called the symmetrical orientation. The resulting complex is denoted G $\alpha$ i_sym + AC5 + ATP.
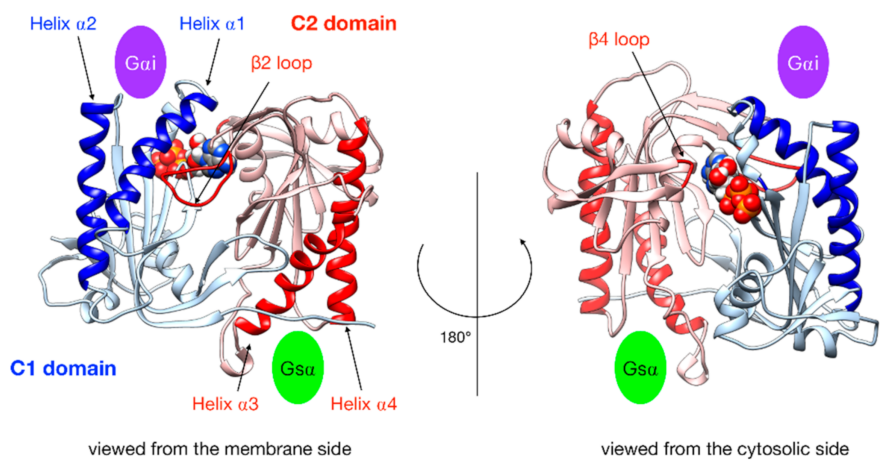

Figure 3. Illustration of the key regions of AC5 catalytic domain structure with bound ATP. The C1 domain is colored blue and the C2 domain is in red, with relevant parts in darker color: the helices of $\mathrm{C} 2$ are involved in the binding of the stimulatory protein $\mathrm{Gs} \alpha$, the helices of $\mathrm{C} 1$ are involved in the binding of the inhibitory protein $G \alpha i$, the $\beta 2$ loop of $C 2$ (left side), and the $\beta 4$ loop of $C 2$ (right side), which bears the catalytic Lysine residue. The green oval indicates the binding site of Gs $\alpha$, and the purple oval indicates the binding site of $\mathrm{G} \alpha \mathrm{i}$. 
A second complex was built by docking the model of AC5 sampled from MD simulation with

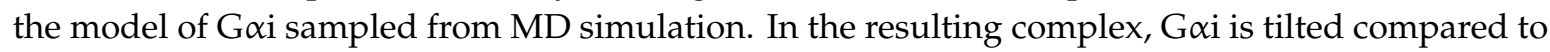
the Gs $\alpha$ orientation with respect to AC5: the G protein is in contact not only with the helix groove, but also with residues on the side of the $\mathrm{C} 1$ domain (see Figure $2 \mathrm{~B}$ ). The resulting complex is denoted Goi_tilted + AC5 + ATP.

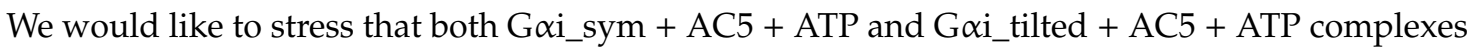
are compatible with the membrane location deduced from the recent full-length structure of AC9 [41], as shown in Figure S2.

Available structures of G $\alpha$ i display different conformations of the N-terminal helix: either protruding from the structure (in 1AGR) or packed onto the structure core (in 1AS3). In the initial model, this helix was packed. During the simulation of $\mathrm{G} \alpha \mathrm{i}$, this helix appeared very mobile. In order to minimize possible bias and to avoid Periodic Boundary Condition problems in the simulations of G $\alpha \mathrm{i}+\mathrm{AC} 5$ complexes, we manually unpacked the N-terminal helix from the structure core after the docking step.

Systems without ATP were also simulated, starting from the same systems after ATP removal. Throughout the study, we compared our results with those obtained in our previous study of AC5 alone and in complex with the activating protein Gs $\alpha$ [25] (see Figure 1, right box).

\subsection{All-Atom Molecular Dynamics Simulations}

Molecular dynamics simulations were performed with the GROMACS 5 package [42-46] using the Amber99SB-ILDN force field for proteins that has been shown to yield an accurate description of many structural and dynamical properties of proteins [47-50]. Side chain protonation states of titratable amino acids were assigned using a value of $\mathrm{pH}=7.4$ with the help of the pdb2pqr software [51]. Capping acetyl and methyl-amino groups were added to the $\mathrm{N}$ and $\mathrm{C}$ termini of both AC5 domains and $G \alpha \mathrm{i}$ to avoid strong artifacts between non-natural termini [23,36]. The four states we study

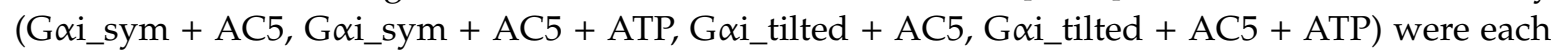
placed in a truncated octahedral box and solvated with TIP3P water molecules [52] to a depth of at least $11 \AA$ A. The solute was neutralized with potassium cations, and then $\mathrm{K}^{+} \mathrm{Cl}^{-}$ion pairs [53] were added to reach a physiological salt concentration of $0.15 \mathrm{M}$. Parameters for ATP and GTP were taken from [54]. The parameters for $\mathrm{Mg}^{2+}$ came from [55]. This new set of parameters was developed to improve the kinetic properties of $\mathrm{Mg}^{2+}$ ions with water and with the phosphate ion, and it was implemented in Amber99. This new set of parameters also provided a better description of the structure of $\mathrm{Mg}^{2+}$-phosphate binding than previous sets (these interactions are naturally important in our simulations in the presence of ATP) [55]. Hence, the combination of Amber 99SB-ILDN and the new set of parameters of $\mathrm{Mg}^{2+}$ ions is currently the best choice to reproduce the dynamics of AC5 and $\mathrm{Gs} \alpha$, and to properly describe the interactions of $\mathrm{Mg}^{2+}$ with AC5 and ATP.

Long-range electrostatic interactions were treated using the particle mesh Ewald method [56,57] with a real-space cutoff of $10 \AA$. We used virtual interaction sites for the hydrogens, and bond lengths were restrained using P-LINCS [45,58], allowing a time step of $4 \mathrm{fs}$ [59]. The translational movement of the solute was removed every 1000 steps to avoid any kinetic energy build-up [60]. After energy minimization of the solvent and equilibration of the solvated system for $10 \mathrm{~ns}$ using a Berendsen thermostat $\left(\tau_{\mathrm{T}}=1 \mathrm{ps}\right)$ and Berendsen pressure coupling $\left(\tau_{\mathrm{P}}=4 \mathrm{ps}\right)$ [61], the simulations were carried out in an NTP ensemble at a temperature of $310 \mathrm{~K}$ and a pressure of 1 bar using a Bussi velocity-rescaling thermostat [62] $\left(\tau_{\mathrm{T}}=1 \mathrm{ps}\right)$ and a Parrinello-Rahman barostat ( $\left.\tau_{\mathrm{P}}=1 \mathrm{ps}\right)$ [63]. Simulations were carried out using typically between 72 and 120 computer cores depending on the system size, which allowed a production rate of about $100 \mathrm{~ns} /$ day. Analysis was carried out on a $1.1 \mu$ s production segment for each simulation, following a $400 \mathrm{~ns}$ equilibration period as in our previous study [25].

\subsection{Analysis of All-Atom Molecular Dynamics Simulations}

We analyzed our all-atom MD simulations using average structures, time-averaged properties such as the RMSD (root mean square deviation), angle between helices, distance between helix axes, 
distance between the ATP $/ \mathrm{Mg}^{2+}$ ion and some key residues, and specific geometrical measurements described below, protein-protein and protein-ligand interface characteristics and, in some cases, residue-by-residue conformational and dynamic properties.

When RMSD distributions indicated the existence of distinct conformations, a cluster analysis was carried out using the gromos algorithm of GROMACS [64], using an RMSD cutoff equal to $1.5 \AA$ on backbone atoms, on the conformations collected in the production phase. Clusters accounting for less than $100 \mathrm{~ns}$ were discarded.

The $\mathrm{C} 1 / \mathrm{C} 2$ interface was characterized using three quantities: the gap volume, the change of accessible surface area upon binding $(\triangle \mathrm{ASA})$, and the Gap index $[65,66]$. The Gap index, defined by the gap volume between two protein chains divided by the interface area, measures the shape complementarity at protein-protein interfaces [66]. The gap volume was computed by the SURFNET software [65], and the interface area was calculated using a local implementation of the Lee and Richards algorithm [67] and the same radii.

In order to characterize $G$ protein binding sites, as in our previous work [25], we computed the angle $\alpha_{\mathrm{C} 2}$ between the pairs of $\alpha$-helices within domain C2 that bind Gs $\alpha$ (termed $\alpha 3$ and $\alpha 4$ in Figure 3) and also the angle $\alpha_{\mathrm{C} 1}$ between the quasi-symmetric pair of helices within domain $\mathrm{C} 1$ (termed $\alpha 1$ and $\alpha 2$ in Figure 3) that binds G $\alpha$ i in the present study. The angles were measured using helical axes derived from the residues that remain in stable $\alpha$-helical conformations throughout the simulations (C1: 408-420 and 468-475, C2: 910-918 and 978-988) as defined in [25]. We also computed the distances between the center of the helices in each domain $\left(\mathrm{d}_{\mathrm{C} 1}\right.$ and $\mathrm{d}_{\mathrm{C} 2}$, respectively).

To characterize protein-protein interfaces, we computed the interface contacts with the python/C code available at https://github.com/MMSB-MOBI/ccmap, using a fixed cutoff of $5 \AA$ between heavy atoms.

To characterize the ATP binding site, we computed two distances between ATP and two key residues for AC5 activity (distance between $\mathrm{O}_{2} \gamma$ and LYS 1065 and between $\mathrm{O}_{2} \alpha$ and ARG 1029) and the distances between ASP 396 and ASP 440 and the two $\mathrm{Mg}^{2+}$ ions [68]. For comparison purposes, we here use the numbering scheme from reference [68]; the corresponding numbering in P84309 is LYS 1245, ARG 1209, ASP 475, and ASP 519.

Data plots were generated with the ggplot2 package in $\mathrm{R}[69,70]$, and structure images are generated with UCSF Chimera [71].

\section{Results}

\subsection{Overview of Simulations}

In the absence of any structural information on the catalytic domains of the enzyme with the inhibiting G-protein subunit G $\alpha$ i, we use a combination of homology modeling, molecular dynamics, and protein-protein docking to get insight on the inhibition mechanism at the molecular level and the impact of the ligand or protein on the conformation and dynamics of AC5.

We have studied the behavior of two molecular species (see Figure 1): AC5 bound to the inhibiting

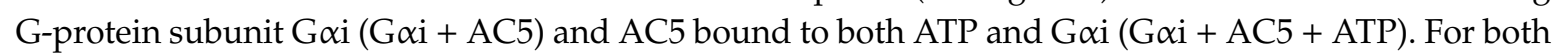
complexes, we considered two different relative conformations: one called Gai_sym + AC5 where the G $\alpha$ i protein has an orientation symmetrical to the Gs $\alpha$ protein in the AC $5+$ Gs $\alpha$ complex, which is

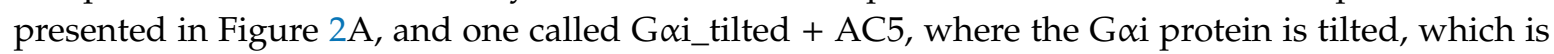
presented in Figure 2B. For each of these species, we generated $1.5 \mu \mathrm{s}$ MD trajectories in an aqueous environment with a physiological salt concentration $(0.15 \mathrm{M} \mathrm{KCl})$. The first $400 \mathrm{~ns}$ of each trajectory were treated as the equilibration of the system, and analysis was carried out only on the remaining $1.1 \mu \mathrm{s}$. In order to understand the effect of G $\alpha \mathrm{i}$ and ATP on AC5, we used the MD simulations for isolated AC5, $\mathrm{AC} 5$ with ATP and two $\mathrm{Mg}^{+}$ions in its active site (AC5 + ATP), AC5 bound to the activating G-protein subunit Gs $\alpha$ (AC5 + Gs $\alpha+$ GTP), and AC5 bound to both ATP and Gs $\alpha$ (AC5 + ATP + Gs $\alpha+$ GTP) obtained in our previous work [25,72]. Data are shown in Tables S1-S5 and in Figures S3-S14. 


\subsection{Stability of Gai + AC5 Complexes in the Presence and in the Absence of ATP}

The two types of complexes behave differently in the presence of ATP. In the G $\alpha$ i_sym + AC5 + ATP simulation, the G $\alpha$ i protein reallocates significantly with respect to AC5 toward the C2 domain, ending in a configuration where it is in contact with the C2 domain; see Figure 4A. The peculiarity of this system is also apparent in the rest of the study and will be commented on later. On the contrary, in the G $\alpha$ i_tilted + AC5 + ATP system, the G $\alpha$ i protein fluctuates around its initial position without significant reallocation, indicating that this complex is very stable; see Figure $4 \mathrm{~B}$. We quantified the fraction of interface contacts between G $\alpha \mathrm{i}$ and AC5 that are conserved throughout the simulation time as well as the total number of interface contacts as a proxy of the interface size. For G $\alpha$ i_sym + AC5 + ATP, the fraction of conserved contacts at the G $\alpha \mathrm{i} / \mathrm{AC} 5$ interface drops rapidly below 25\%, while the total number of contacts increases significantly (see Figure S3). In G $\alpha$ i_tilted + AC5 + ATP, 50 to 75\% of the initial contacts are conserved during the simulation time, and the total number of contacts also tends to increase, although less dramatically (Figure S3). In the absence of ATP, both systems maintain between 50 and $75 \%$ of their initial contacts, with more moderate reallocation and variation in terms of contact number (see Figures S3 and S4).

A Gai_sym+AC5+ATP

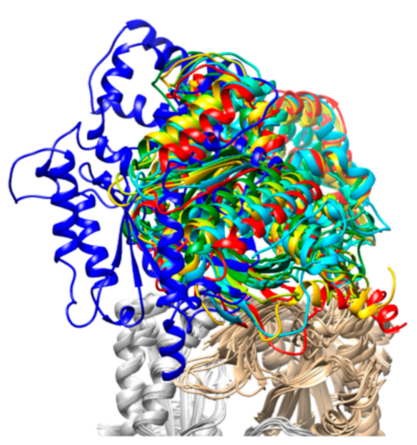

B Gai_tilted+AC5+ATP

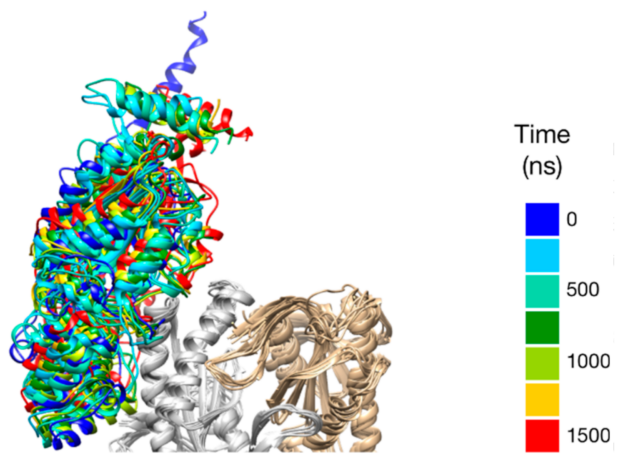

Figure 4. Snapshots of the G $\alpha \mathrm{i}+\mathrm{AC} 5+$ ATP complexes observed during the simulations, viewed from the membrane side. G $\alpha$ i structures extracted every $250 \mathrm{~ns}$ are colored on a rainbow scale from blue to red. The $\mathrm{C} 1$ domain of $\mathrm{AC} 5$ is colored in gray and the $\mathrm{C} 2$ domain in beige.

\subsection{Impact of Gai on AC5 + ATP}

We begin by considering the global impact of G $\alpha$ i on AC5 + ATP by computing the RMSD on backbone atoms separately on each AC5 domain. RMSD calculations with respect to the average MD structure of each AC5 domain show that G $\alpha$ i binding has a significant effect on both the structure and the dynamics of the enzyme (see Table S1 and Figure S5 where in order to allow comparison with the results obtained in our previous study [25], values for AC5, AC5 + ATP, AC5 + Gs $\alpha$, and AC5 + ATP + Gs $\alpha$ were also included). On the one hand, the domain $C 1$ is slightly rigidified by the binding of G $\alpha$ i. On the other hand, the C2 domain visits several conformational substates involving the ATP binding pocket ( $\beta 2$ loop and $\beta 4$ loop) in the G $\alpha$ i_sym + AC5 complex (see Figure 5). These two substates also lead to two different substates for ATP (see Figure S6) which is more mobile, increasing the average RMSD from $0.6 \AA$ for AC5 + ATP to $0.9 \AA$ for G $\alpha$ i_sym + AC5 + ATP (see Table S1 and Figure S7). In the case of G $\alpha$ i_tilted + AC5 + ATP, the C2 domain visits a specific substate close to the one sampled in AC5 + ATP where the mobility of ATP is unchanged due to the presence of Gai. 

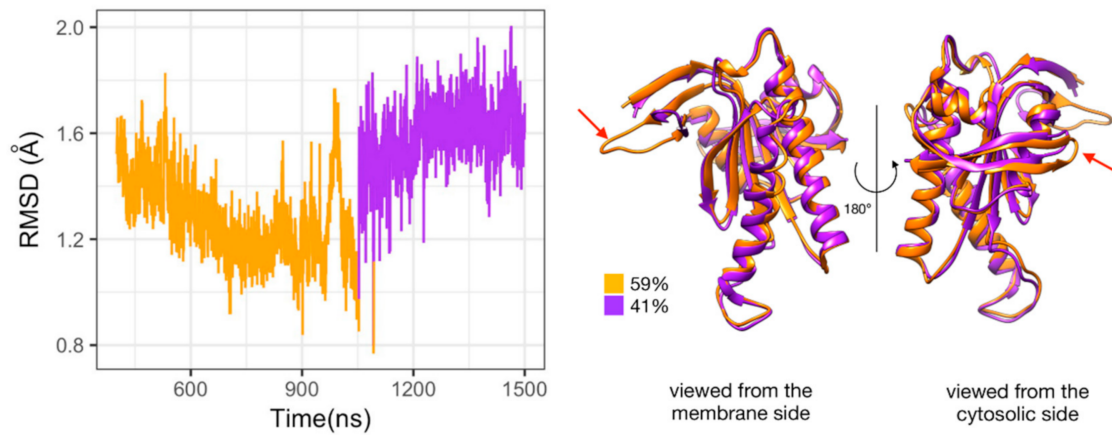

Figure 5. Substates of domain C2 observed during the simulation of G $\alpha$ i_sym + AC5 + ATP complex. Left side: Root mean square deviation (RMSD) time series for the C2 domain, colored according to cluster membership. Right: structures closest to the center of each cluster, and relative size of each cluster as percentages. Prominent structural changes are indicated by red arrows.

The presence of several substates upon binding of G $\alpha \mathrm{i}$ is in contrast with the stabilization of a specific substate upon the binding of ATP and/or Gs $\alpha$. Indeed, in our previous work, we observed that the $\mathrm{C} 2$ domain can visit several substates when AC5 is isolated, and the presence of either ATP alone or ATP and Gs $\alpha$ stabilizes two distinct substates. In the former case, a single substate for the $\beta 2$ loop is selected (the longest-lived substate in isolated AC5) in a closed conformation. In the latter, an opening of loop $\beta 2$ away from the active site is observed. The selection of a specific substate is correlated to the mobility of ATP and its reactivity [25].

Despite the decrease in flexibility of the C2 domain, which is also observed when Gs $\alpha$ is bound to AC5 + ATP, in the presence of Gai, ATP is still rather mobile (see Table S1), and for Gai_sym + AC5 + ATP, an increase in mobility is observed: this impact is opposite to the one observed in AC5 + ATP + Gs $\alpha$, where a higher stability of ATP is observed (average RMSD equal to $0.3 \AA$ ). In both simulations in the presence of ATP, the interactions between the terminal phosphate group of ATP and LYS 1065 (belonging to loop $\beta 2$ ) and the interactions between the penultimate phosphate and ARG 1029, a key functional residue, are absent (see Figure S8): the arginine side chain is separated from its target oxygen atom by roughly $9 \AA$. Moreover, it is known that ATP has stronger interactions with the $\mathrm{C} 1$ domain via its associated two $\mathrm{Mg}^{2+}$ ions, notably with residues ASP 396 and ASP 440. For G $\alpha$ i_tilted + AC5 + ATP, these interactions are stable and are not affected by the presence of G $\alpha \mathrm{i}$. On the contrary, for G $\alpha$ i_sym + AC5 + ATP, these interactions are absent, justifying the increase of ATP mobility (see Table S3).

G $\alpha$ i binding also turns out to have more global effects on AC5 + ATP. First, the angles and the distance between the pairs of $\alpha$-helices in both AC5 domains are modified. In G $\alpha$ i_sym + AC5 + ATP, the angle between the helices $\alpha 1$ and $\alpha 2$ in domain $\mathrm{C} 1$ is significantly reduced (by $9^{\circ}$, see Table S1) and the angle between the helices $\alpha 3$ and $\alpha 4$ in domain C2 is slightly increased (by $4^{\circ}$ ). In G $\alpha$ i_tilted + AC5 + ATP, an opposite effect is observed: the angle between the helices $\alpha 1$ and $\alpha 2$ in domain $\mathrm{C} 1$ is hardly affected (increased by $1^{\circ}$, see Table S1 and Figure S9), and the angle between the helices $\alpha 3$ and $\alpha 4$ in domain $\mathrm{C} 2$ is slightly decreased (by $3^{\circ}$ ). In addition, the distance between the C2 helices in Gai_tilted + AC5 + ATP complexes is maintained around $13 \AA$, whereas our earlier results indicate that it is around $16 \AA$ in the AC5 + ATP + Gs $\alpha$ complex (see Table S1 and Figure S10). In both simulations, the $\mathrm{C} 1 / \mathrm{C} 2$ interface remains mostly as tight as in isolated AC5 + ATP (gap index from $2.8 \AA$ for AC5 + ATP to $2.9 \AA$ once G $\alpha$ i is bound, Table S1 and Figure S11) involving a movement of helix $\alpha 3$ (see Figure 6). In terms of flexibility, G $\alpha$ i binding mainly flexibilizes the binding site region of ATP in AC5, although it also decreases the flexibility of the C-terminals of helices $\alpha 1$ and $\alpha 3$ (see Figure 7A). 
A
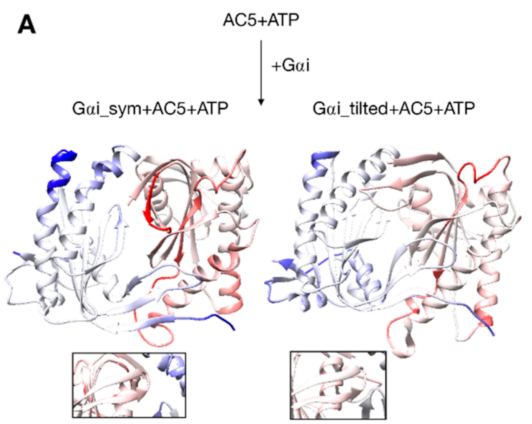

B

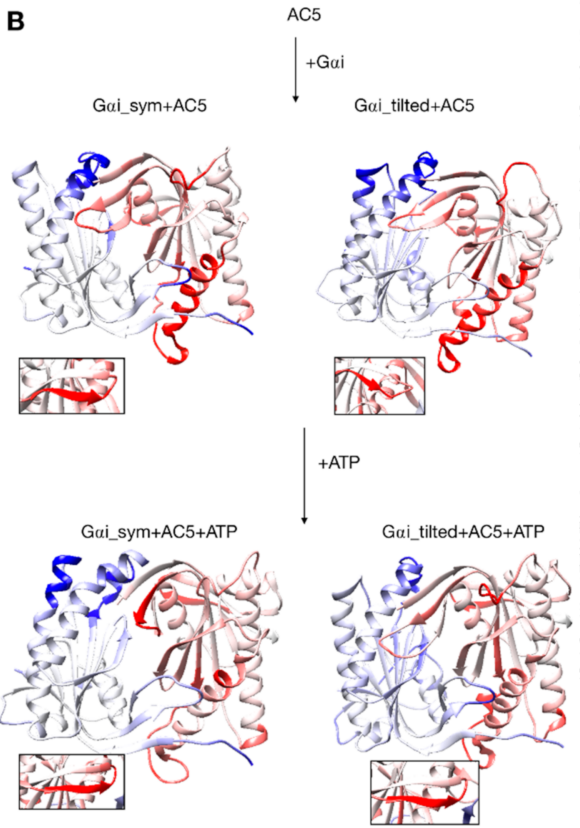

Figure 6. Changes in conformation induced by G $\alpha$ i protein. (A) scenario where ATP is already bound

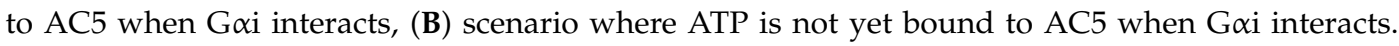
More intense colors (blue for domain $\mathrm{C} 1$ and red for domain $\mathrm{C} 2$ ) correspond to larger movements compared to the preceding structure (i.e., AC5 + ATP for A and AC5 and AC5 + G $\alpha$ i for B) on a scale of 0 to $4 \AA$. The insets display the $\beta 4$ loop.
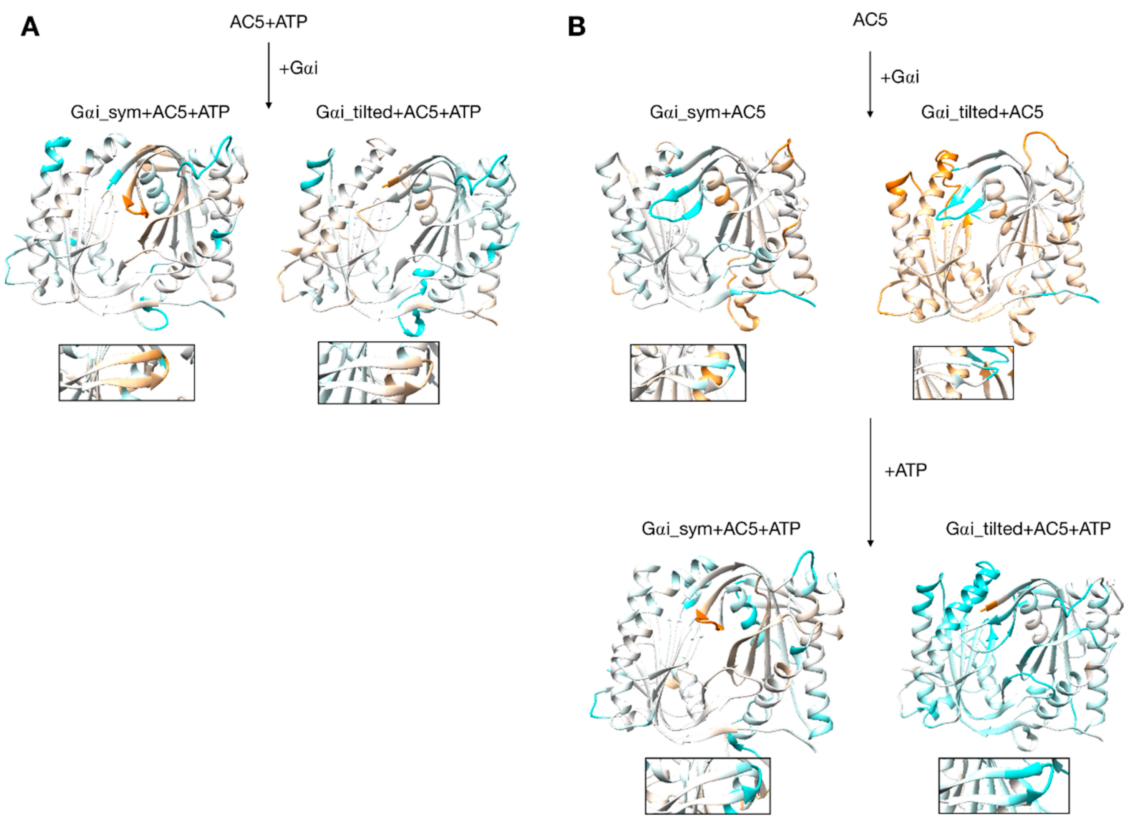

Figure 7. Changes in flexibility induced by G proteins. (A) Scenario where ATP is already bound to AC5

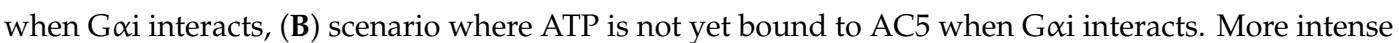
colors (orange for increased flexibility and cyan for decreased flexibility) correspond to differences with respect to the preceding structure on a scale of -1.2 to $+1.2 \AA$. The insets display the $\beta 4$ loop.

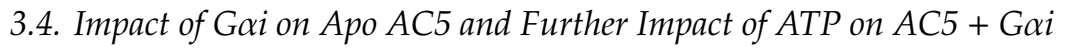

Although it seems probable that ATP is already bound to AC5 based on our previous study [25], we also consider the scenario where ATP is not already present when Gai binds on AC5. We begin by

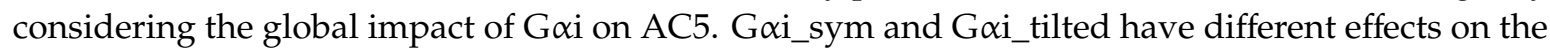


C1 domain of AC5: Gai_sym slightly rigidifies it as attested by the RMSD calculation (Table S1 and Figure S5), whereas G $\alpha$ i_tilted flexibilizes it, and this flexibility concerns the binding helices $\alpha 1$ and $\alpha 2$ (Figure 7B). For the C2 domain, in both G $\alpha$ i_sym + AC5 and G $\alpha$ i_tilted + AC5 complexes, the $\beta 2$ loop and the $\beta 4$ loop are rigidified upon the addition of $\mathrm{G} \alpha \mathrm{i}$ (see Figure $7 \mathrm{~B}$ ). Their conformations slightly

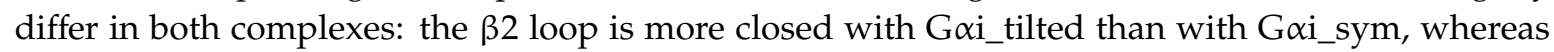

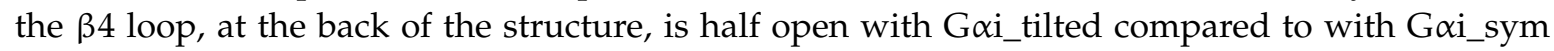
(see Figure S12). In G $\alpha$ i_sym + AC5, the C2 domain visits several conformational substates involving the helices $\alpha 3$ and $\alpha 4$ (see Figure S13).

The conformation of the binding helices in both AC5 domains is significantly altered by Gai.

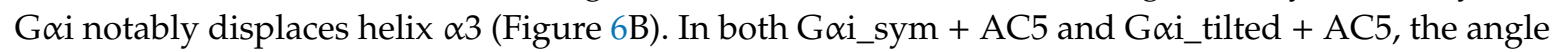
between the helices $\alpha 1$ and $\alpha 2$ in domain C1 is significantly increased (by $24^{\circ}$ and $19^{\circ}$; see Table S1 and Figure S9). On the contrary, the angle between the helices $\alpha 3$ and $\alpha 4$ in domain C2 is significantly decreased (by $7^{\circ}$ and $12^{\circ}$ ), and the helices are also closer to each other (Table S1 and Figure S10). The C1/C2 interface remains as tight as in isolated AC5, in contrast with what we observed previously with the binding of Gs $\alpha$, which resulted in a looser C1/C2 interface (gap index equal to $3.8 \AA$, see Table S2 and Figure S11).

In the scenario where ATP is not already bound to AC5 when G $\alpha$ i interacts, we can also analyze the effect of ATP addition on the pre-formed G $\alpha \mathrm{i}+\mathrm{AC} 5$ complex. As shown in Figure 7B, the addition

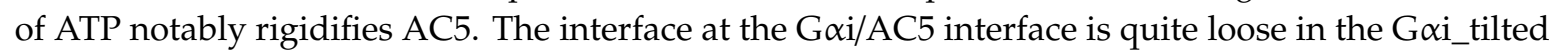
+ AC5 complex (gap index equal to $4.6 \AA$, see Table S2), and the addition of ATP tends to tighten this interface (gap index equal to $4.2 \AA$ ). On the contrary, in the G $\alpha$ i_sym + AC5 complex, the initial G $\alpha \mathrm{i} / \mathrm{AC} 5$ interface is made more loose by the addition of ATP (gap index from $3.2 \AA$ in G $\alpha \mathrm{i}+\mathrm{AC} 5$ to $5.4 \AA$ when ATP is bound), which is probably due to the change of interface as observed by the losing of $75 \%$ of the native contacts by adding ATP (see Figure S3). By comparison, in the AC5 + Gs $\alpha$ complex, the gap index decreased from 3.2 to $2.7 \AA$ upon ATP addition (Table S2). Despite the variation of the values of gap index for the G $\alpha \mathrm{i} / \mathrm{AC} 5$ interface upon the binding of ATP, all the values are typical of obligate protein-protein interfaces [73].

\section{Discussion}

As already observed in our previous work [25], microsecond-scale simulations are necessary to investigate the allosteric coupling existing within AC5 and the effect of the binding of G $\alpha$ i. Similarly to van Keulen and Rothlisberger [28], we studied the scenario where G $\alpha$ i binds to AC5 in the absence of ATP, but we could not exclude the possibility that ATP is bound on AC5 when Gai binds, based on our previous work [25]. Due to the lack of structural information on the complex between G $\alpha \mathrm{i}$ and AC5, we generated docked models using CLUSPRO, which is a server that achieves good results in the blind community-wide assessment CAPRI [74]. In order to reduce possible structural bias, we used as input isolated protein models sampled from MD simulations and integrated putative interface residues [24] in the form of a soft constraint by specifying attractive residues for the docking

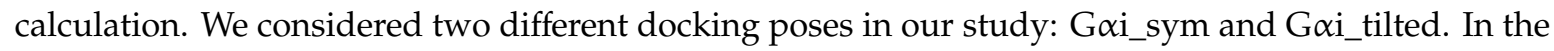
former, the G $\alpha$ i protein is bound to AC5 in a symmetrical fashion compared to what is known for the $\mathrm{AC} 5+\mathrm{ATP}+\mathrm{Gs} \alpha$ complexes (Figure 2A) and similar to the model postulated by Dessauer et al. [24]. In the latter, the $\mathrm{G} \alpha \mathrm{i}$ protein is rotated and tilted onto the $\mathrm{C} 1$ domain (Figure $2 \mathrm{~B}$ ). Both complexes stay bound during the simulations, but a greater stability is obtained for the G $\alpha$ i_tilted configuration (see Figure 4), suggesting more biological relevance. In the case where G $\alpha$ i binds on AC5 + ATP in

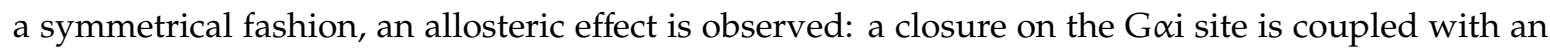
opening on the Gs $\alpha$ site and an opening of the $\beta 2$ loop of C2, as observed for the binding of Gs $\alpha$. Despite that, this conformation seems to be less likely because the complementarity is very low and the interface is unstable, as already mentioned above. On the contrary, the G $\alpha$ i_tilted configuration is also very similar to the one reported by van Keulen and Rothlisberger in their recent work where they studied the complex between myristoylated G $\alpha$ i and AC5 in the absence of ATP [28], although 
the starting conformations of AC5 and G $\alpha \mathrm{i}$ are quite different, as well as the docking procedures. Given the inherent complexity of protein-protein docking, such convergence toward similar models despite different approaches is an additional support to the biological relevance of this model.

On the experimental side, several mutations on AC5 are known to impact its regulation by Gai: ASP411, MET414, THR415, and ASP418 in helix $\alpha 1$, LEU472 and VAL476 in helix $\alpha 2$, and VAL479 in the loop connecting helix $\alpha 2$ to a $\beta$-strand [24] (see Table S4 for the numbering scheme). In our simulations of the G $\alpha$ i_tilted + AC5 complex, these residues are remarkably persistent, i.e., present more than $80 \%$ of the time at the interface between AC5 and G $\alpha$ i with and without ATP; see Table S4. Not only are these residues persistent at the interface, but they also form stable interactions with particular side chains on G $\alpha$ i belonging to the switch I, switch II and switch III regions [75] (see Table S5 and Figure S14). The high stability of such interactions observed in our MD simulations suggest a functional role for these residues, which is consistent with the fact that the mutations affecting them have an impact on the regulation of AC5 by Gai, as observed experimentally [24].

The binding of $\mathrm{G} \alpha \mathrm{i}$ slightly rigidifies the $\mathrm{C} 2$ domain in all simulations, and an opening of the $\mathrm{G} \alpha \mathrm{i}$ binding site is coupled with a closure of the Gs $\alpha$ binding site, in particular in the Gai_tilted + AC5 complexes with and without ATP, as observed in van Keulen and Rothlisberger's simulation [28]. As in the case of the AC5 + Gs $\alpha$ complex, ATP stabilizes the G $\alpha \mathrm{i} / \mathrm{AC} 5$ interface when G $\alpha \mathrm{i}$ is tilted, whereas the latter is less complementary. All these changes involve coupling through AC5 over distances of tens of angstroms.

Turning now to the enzymatic function of AC5, it is known that specific hydrogen bonds between AC5 and ATP play an important role in the production of cAMP from ATP, as already shown in hybrid QM/MM free energy calculations, notably the hydrogen bond between the highly conserved ARG 1029 and the $\alpha$ phosphate group of ATP [68]. The present simulations show that this interaction is not formed upon the binding of G $\alpha$ i and other ones are lost (for example between LYS 1065 and ATP, see Figure S8), when on the contrary, it is formed upon the binding of Gs $\alpha$ (see Figure 8). This structural effect of G $\alpha \mathrm{i}$, which stabilizes the active site of AC5 in a state that prevents ATP to form the required interactions for catalysis, provides a structural basis for the enzymatic inhibition of AC 5 by G $\alpha \mathrm{i}$ observed experimentally [24].

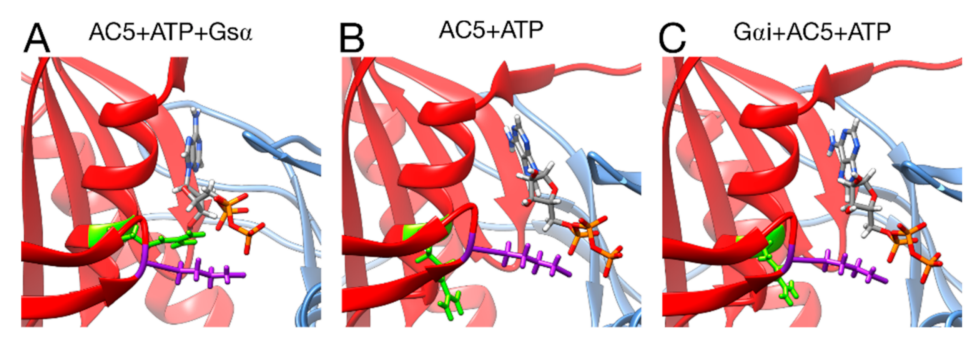

Figure 8. Interactions between ATP and key residues in different complexes. (A) active AC5 + ATP + Gs $\alpha,($ B $)$ inactive AC5 + ATP complex, (C) inactive G $\alpha$ i_tilted + AC5 + ATP complex. Key residues of C2 are shown as sticks and colored in purple (LYS 1065) and green (ARG 1029). The C1 and C2 subunits of AC5 are colored blue and red, respectively. For clarity, the region 394-428 of C1 is omitted from the representation.

The binding of G $\alpha \mathrm{i}$ inhibits AC5 by increasing the flexibility of the active site allowing a high mobility of ATP without changing the complementarity of the $\mathrm{C} 1 / \mathrm{C} 2$ interface. It was proposed previously [24] that the inhibitory effect of G $\alpha$ i might be due to the disruption of the active site of AC5 or a decrease of the affinity between $\mathrm{C} 1$ and $\mathrm{C} 2$ domains. Our simulation study is in line with this first hypothesis. The further inactivation of AC $5+$ ATP by G $\alpha$ i does not exclude the possibility that ATP is bound to AC5 during the inhibition process, and it may have a role in the tight regulation of the enzyme.

Based on our previous study [25] and the current one, we can speculate on the regulation mechanism of AC5 by considering only the G $\alpha$ i_tilted configuration. In the absence of ATP, based on 
our simulations, only Gs $\alpha$ could interact with AC5, due to the close conformation of the binding site on the $\mathrm{C} 1$ domain. However, we cannot exclude the existence of $\mathrm{G} \alpha \mathrm{i}+\mathrm{AC} 5$ based on our study. The binding of ATP induces an opening of the angle between the pair of $\alpha$-helices of domain $\mathrm{C} 1\left(\alpha_{\mathrm{C} 1}\right)$ and a closure of the pair of $\alpha$-helices of domain C2 ( $\left.\alpha_{\mathrm{C} 2}\right)$, which have a similar value close to the one observed upon the binding of Gs $\alpha$. If G $\alpha$ i binds first AC5 + ATP, the closure of the angle $\alpha_{\mathrm{C} 2}$ does not allow the binding of Gs $\alpha$, and the ATP pocket remains close. When G $\alpha$ i dissociates from AC5 + ATP, AC5 undergoes a conformational change that allows the binding of Gs $\alpha$. On the contrary, if Gs $\alpha$ binds AC5 first, the enzyme is active thanks to the stabilization of ATP in its pocket and the formation of specific hydrogen bonds and cycles between AC5 + ATP + Gs $\alpha$ (favorable to G $\alpha$ i binding) and AC5 + Gs $\alpha$ (unfavorable to G $\alpha$ i binding). If Gs $\alpha$ dissociates, after cAMP release, AC5 is in an apo conformation, and there is no need for further inhibition. If Gs $\alpha$ dissociates from AC5 + ATP, then the conformation of AC5 becomes accessible to G $\alpha$ i for the inhibition. Another possibility is that due to the open conformation of the binding site on the C1 domain, G $\alpha$ i can bind to the AC5 + ATP + Gs $\alpha$ complex to form a ternary complex, whose existence is still unknown.

\section{Conclusions}

We perform all-atom molecular dynamics simulations in an attempt to better understand the regulation of adenylyl cyclase, which is a key enzymatic player in cellular signaling cascades. Microsecond-scale simulations of the G-protein subunit G $\alpha$ i bound to the cytoplasmic domains of adenylyl cyclase in the presence and in the absence of ATP in two different conformations help to better understand some features of this important signal transmission protein, since no structural information on this complex is available. They notably provide information on a single, non-chimeric adenylyl cyclase isoform, AC5, which is bound to the inhibitory G-protein in the presence and in the absence of ATP.

The simulations show that protein binding creates significant changes in the structure and in flexibility throughout $\mathrm{AC} 5$ and due to a strong allosteric coupling existing within AC5 in a different fashion than the stimulatory G-protein and ATP. They provide data that help to explain the inhibition

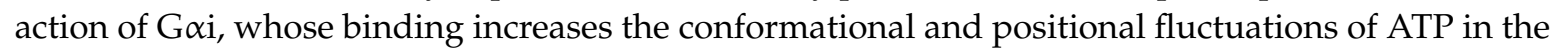
active site of AC5 and its flexibility by moving away the key residues involved in the enzymatic reactions.

Our results also show that $\mathrm{G} \alpha \mathrm{i}$ binding to the $\mathrm{C} 1$ domain does not impact $\mathrm{C} 1 / \mathrm{C} 2$ interface complementarity, flexibilizes the $\mathrm{C} 1$ domain, and significantly closes the angle between the $\mathrm{C} 2 \alpha$-helices

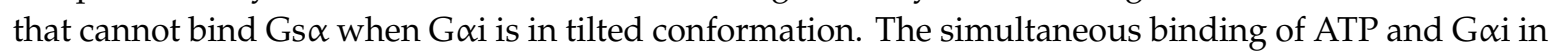
a tilted conformation at the $\mathrm{AC} 5$ interface results in a rigidification of the $\mathrm{C} 2$ domain, without affecting the $\mathrm{C} 1 / \mathrm{C} 2$ interface complementarity, and a slight increase of the angle between the $\mathrm{C} 2 \alpha$-helices. Hence, G $\alpha$ i has an important impact on AC5 dynamics, and its effects are enhanced when ATP binds by increasing the conformational freedom of the bound ligand, thus putting it in an unfavorable configuration within its binding site and not allowing the establishment of key interactions between ATP and AC5, which therefore leaves AC5 completely inactive.

Our simulations also show that ATP has a crucial role in the regulation of AC5, and we cannot exclude the presence of ATP during the inhibition. Our previous simulations already showed that ATP binding could influence the binding of the inhibitory G-protein subunit $\alpha \mathrm{i}$ at the C1 domain. Here, we propose that the presence of ATP is needed to induce the competition between Gs $\alpha$ and G $\alpha$ i to tightly regulate AC5. However, based on our results, we cannot exclude the existence of the G $\alpha \mathrm{i}+\mathrm{AC} 5+\mathrm{ATP}+\mathrm{Gs} \alpha$ complex and the G $\alpha \mathrm{i}+\mathrm{AC} 5+\mathrm{Gs} \alpha$ complex. For the latter, other molecular dynamic studies of this hypothetical ternary complex concluded that if it exists, it would be inactive $[29,30]$. Further studies have to be conducted to shed light on this point.

Supplementary Materials: The following are available online at http://www.mdpi.com/2218-273X/10/9/1330/s1, Figure S1: G $\alpha \mathrm{i}+$ AC5 + ATP complexes, viewed from the membrane side. G $\alpha \mathrm{i}$ is colored in purple, AC5 is colored in blue (C1 domain) and red (C2 domain). In green, residues labeled as "attractive" for the docking: residues 484-486 and 554-558 in AC5 (numbered according to P84309), which are at the entry of the binding site suggested 
by mutations in [24], and residues 209-216 in Gai (numbered according to P08752), which are in the switch II region (201-221) involved in the binding [24]. The switch I and switch III regions [75] are indicated by arrows. Figure S2. Superimposition of the G $\alpha \mathrm{i}+\mathrm{AC} 5+$ ATP complexes simulated in this study superimposed with the full-length structure of AC9 bound to Gs $\alpha$ (PDB structure 6R3Q [41]). Blue: C1 domain of AC5, red: C2 domain of AC5, purple: G $\alpha$ i, gray: full-length AC9 bound with Gs $\alpha$. Membrane location from [41]. Figure S3. Number of contacts at the AC5/G $\alpha$ i interface. Contacts are defined using a $5 \AA$ cut-off between heavy atoms. Top row: Fraction of initial contacts $(t=0)$ that are maintained as a function of time. Bottom row: total number of contacts between AC5 and G $\alpha \mathrm{i}$; dashed horizontal lines indicate the number of contacts at $t=0$. Figure S4: Snapshots of the G $\alpha \mathrm{i}+\mathrm{AC} 5$ complexes observed during the simulations without ATP, viewed from the membrane side. Structures extracted every $250 \mathrm{~ns}$ are colored on a rainbow scale from blue to red. The C1 domain of AC5 is colored in gray, and the C2 domain is colored in beige. Figure S5: RMSD distribution for C1 and C2 domains, with respect to average structures, in the simulations with and without ATP. Data for AC5 and AC $5+$ Gs $\alpha$, with and without ATP, taken from $[25,72]$. All the densities are computed with ggplot2 in $\mathrm{R}[66,67]$, using default parameters (Gaussian kernel density estimator and 512 equally spaced points). Figure S6: Substates of ATP and C2 observed in the Gai_sym + AC5 + ATP simulation. A: Structural clusters obtained using gromos (cutoff $=1.5 \AA$ on backbone atoms for $\mathrm{C} 2$ and cutoff $=1 \AA$ for ATP), B: average structures viewed from the membrane side, C: close-up view on the $\beta 4$ loop, from the cytoplasmic side. The average structures are colored in gray $(400 \mathrm{~ns}<\mathrm{T}<1100 \mathrm{~ns})$ and yellow (1200 ns < T < $1500 \mathrm{~ns}$ ). Figure S7: RMSD distribution for ATP. Data for AC5 + ATP and AC5 + ATP + Gs $\alpha$ taken from $[25,39]$. Figure S8: Distance between ATP and key residues of C2. Data for AC5 + ATP and AC5 + ATP + Gs $\alpha$ are from our previous study [25,72]. ATP/ARG1029: distance between the O2 $\alpha$ of ATP and the center of mass of terminal hydrogen atoms, which are covalently bound to $\mathrm{N} \varepsilon$ of ARG 1029. ATP/LYS1065: distance between the $\mathrm{O} 2 \gamma$ of ATP and the center of mass of the terminal hydrogen atoms, which are covalently bound to N $\zeta$ of LYS 1065. Figure S9: Distribution of the angles between helix axes (helix $\alpha 1$ and helix $\alpha 2$ in C1, helix $\alpha 3$ and helix $\alpha 4$ in C2, see Figure 3). Data for AC5 and AC5 + Gs $\alpha$, with and without ATP, taken from [25,72]. Figure S10: Distribution of the distance between helix axes (helix $\alpha 1$ and helix $\alpha 2$ in C1, helix $\alpha 3$ and helix $\alpha 4$ in C2, see Figure 3). Data for $\mathrm{AC} 5$ and AC5 + Gs $\alpha$, with and without ATP, taken from [25,39]. Figure S11: Distribution of the C1/C2 interface gap index. Data for AC5 and AC5 + Gs $\alpha$, with and without ATP, taken from [25,72]. Figure S12. Local comparison of

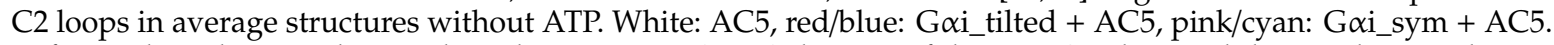
Left panel: $\beta 2$ loop, right panel: $\beta 4$ loop. Figure S13. Substates of domain C2 observed during the simulation of Gai_sym + AC5 complex, without ATP. Left: RMSD time series for the C2 domain, colored according to cluster membership. Right: Structures closest to the center of each cluster, and the relative size of each cluster as percentages. Prominent structural changes are indicated by red arrows. Figure S14. The persistent interface residues in Gai_tilted + AC5 + ATP simulation. The C1 domain is colored in blue, the C2 domain is colored in red, $\mathrm{G} \alpha \mathrm{i}$ is colored in purple. Important residues on $\mathrm{C} 1$ from reference [24] that are persistent at the interface with $\mathrm{G} \alpha \mathrm{i}$ are shown as sticks, which are colored in orange and labeled with a residue numbering from [24]. Residues on G $\alpha \mathrm{i}$ that form stable interactions from Table S5 are shown in sticks and colored in pink (switch I), yellow (switch II), and cyan (switch III). Table S1. Average and standard deviation of backbone RMSD for the C1 and C2 domains of $\mathrm{AC} 5$, angle between helices $(\alpha \mathrm{C} 1$ and $\alpha \mathrm{C} 2)$, distance between helices axes (dC1 and $\mathrm{dC} 2)$, Gap index for the interface C1/C2, RMSD for ATP. a: data in italics are from our previous study $[25,39]$. Table S2: Mean values and

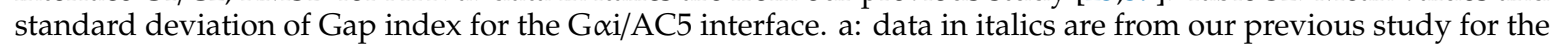
Gap index for the Gs $\alpha / A C 5$ interface [25,72]. Table S3. Distance between $\mathrm{Mg}^{2+}$ ions and the aspartate residues (ASP 396 and ASP 440 in C1 domain). Table S4. Persistence of residues of AC5 mutated in reference [24] at AC5/G $\alpha$ i interface in our simulations. The fraction of time each residue is part of the AC5/G $\alpha$ i interface is shown as a percentage, and the number of contacting residues on $\mathrm{G} \alpha \mathrm{i}$ is between parentheses. Interface residues are defined using a $5 \AA$ cut-off between heavy atoms. Residues in italics have limited or no impact on the binding. ${ }^{1}$ : For the sake of comparison, residue numbering refers to reference [24]; the corresponding numbers in Uniprot sequence P84309 are between parentheses. ${ }^{2}$ : In Ref. [24], the double mutant M414/T415 was studied; hence, we monitor the presence of M414 or T415. Table S5. Stable interactions between key residues of AC5 from reference [24] and residues of $\mathrm{G} \alpha \mathrm{i}$. Interactions that appear more than $50 \%$ of the time in each simulation are listed with the corresponding fraction of time between parentheses. Interactions are defined using a $5 \AA$ cut-off between heavy atoms. Interactions that are common to simulations with and without ATP for a given system are highlighted in bold. For the sake of comparison, AC5 residue numbering refers to reference [24]; the corresponding numbers in

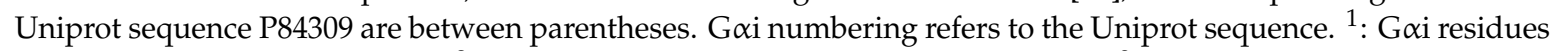
belonging to the switch I region, ${ }^{2}$ : G $\alpha$ i residues belonging to the switch II region, ${ }^{3}$ : G $\alpha$ i residues belonging to the switch III region [75].

Author Contributions: Conceptualization, E.F. and J.M.; validation, E.F. and J.M.; formal analysis, T.-M.A. and J.M.; investigation, E.F. and. T.-M.A.; resources, E.F. and J.M.; data curation, E.F. and J.M.; writing-original draft preparation, E.F. and J.M.; writing-review and editing, E.F. and J.M.; supervision, E.F. and J.M.; funding acquisition, E.F. and J.M. All authors have read and agreed to the published version of the manuscript.

Funding: This project/research has received funding from the European Union's Horizon 2020 Framework Programme for Research and Innovation under the Specific Grant Agreement No. 720270 (Human Brain Project SGA1) and the Specific Grant Agreement No. 785907 (Human Brain Project SGA2). 
Acknowledgments: We thank Alexis Michon and Samuel Bosquin (UMS 3760, Institut de Biologie et Chimie des Protéines, Lyon, France) for technical assistance and hardware support. We wish to acknowledge GENCI for a generous allocation of computer time on the CINES supercomputer OCCIGEN (Grant 2016-c201607758, Grant 2017-A0020707585 and Grant 2018-A0040710357).

Conflicts of Interest: The authors declare no conflict of interest.

\section{References}

1. Hanson, M.A.; Stevens, R.C. Discovery of New GPCR Biology: One Receptor Structure at a Time. Structure 2009, 17, 8-14. [CrossRef] [PubMed]

2. Hardman, J.G.; Robison, G.A.; Sutherland, E.W. Cyclic Nucleotides. Annu. Rev. Physiol. 1971, 33, $311-336$. [CrossRef] [PubMed]

3. Andersson, R.; Nilsson, K. Cyclic AMP and Calcium in Relaxation in Intestinal Smooth Muscle. Nat. New Biol. 1972, 238, 119-120. [CrossRef] [PubMed]

4. DeMaria, S.; Ngai, J. The cell biology of smell. J. Cell Biol. 2010, 191, 443-452. [CrossRef] [PubMed]

5. Davis, R.L.; Cherry, J.; Dauwalder, B.; Han, P.-L.; Skoulakis, E.M.C. The cyclic AMP system andDrosophila learning. In Signal Transduction Mechanisms; Springer: Berlin, Germany, 1995; pp. 271-278.

6. Kandel, E.R. The Molecular Biology of Memory Storage: A Dialogue Between Genes and Synapses. Science 2001, 294, 1030-1038. [CrossRef]

7. Wu, Z.L.; Thomas, S.A.; Villacres, E.C.; Xia, Z.; Simmons, M.L.; Chavkin, C.; Palmiter, R.D.; Storm, D.R. Altered behavior and long-term potentiation in type I adenylyl cyclase mutant mice. Proc. Natl. Acad. Sci. USA 1995, 92, 220-224. [CrossRef]

8. Kamenetsky, M.; Middelhaufe, S.; Bank, E.M.; Levin, L.R.; Buck, J.; Steegborn, C. Molecular Details of cAMP Generation in Mammalian Cells: A Tale of Two Systems. J. Mol. Biol. 2006, 362, 623-639. [CrossRef]

9. Sunahara, R.K.; Dessauer, C.W.; Gilman, A.G. Complexity and Diversity of Mammalian Adenylyl Cyclases. Annu. Rev. Pharmacol. Toxicol. 1996, 36, 461-480. [CrossRef]

10. Sunahara, R.K. Isoforms of Mammalian Adenylyl Cyclase: Multiplicities of Signaling. Mol. Interv. 2002, 2, 168-184. [CrossRef]

11. Krupinski, J.; Coussen, F.; Bakalyar, H.; Tang, W.; Feinstein, P.; Orth, K.; Slaughter, C.; Reed, R.; Gilman, A. Adenylyl cyclase amino acid sequence: Possible channel- or transporter-like structure. Science 1989, 244, 1558-1564. [CrossRef]

12. Tang, W.-J.; Gilman, A.G. Adenylyl cyclases. Cell 1992, 70, 869-872. [CrossRef]

13. Tesmer, J.J.; Sunahara, R.K.; Johnson, R.A.; Gosselin, G.; Gilman, A.G.; Sprang, S.R. Two-Metal-Ion Catalysis in Adenylyl Cyclase. Science 1999, 285, 756-760. [CrossRef] [PubMed]

14. Jacobowitz, O.; Chen, J.; Premont, R.T.; Iyengar, R. Stimulation of specific types of Gs-stimulated adenylyl cyclases by phorbol ester treatment. J. Biol. Chem. 1993, 268, 3829-3832.

15. Gilman, A.G. G proteins and regulation of adenylyl cyclase. Biosci. Rep. 1995, 15, 65-97. [CrossRef] [PubMed]

16. Rasmussen, S.G.F.; DeVree, B.T.; Zou, Y.; Kruse, A.C.; Chung, K.Y.; Kobilka, T.S.; Thian, F.S.; Chae, P.S.; Pardon, E.; Calinski, D.; et al. Crystal structure of the $\beta 2$ adrenergic receptor-Gs protein complex. Nature 2011, 477, 549-555. [CrossRef]

17. Nygaard, R.; Zou, Y.; Dror, R.O.; Mildorf, T.J.; Arlow, D.H.; Manglik, A.; Pan, A.C.; Liu, C.W.; Fung, J.J.; Bokoch, M.P.; et al. The dynamic process of $\beta(2)$-Adrenergic receptor activation. Cell 2013, 152, 532-542. [CrossRef]

18. Patel, T.B.; Du, Z.; Pierre, S.; Cartin, L.; Scholich, K. Molecular biological approaches to unravel adenylyl cyclase signaling and function. Gene 2001, 269, 13-25. [CrossRef]

19. Sadana, R.; Dessauer, C.W. Physiological roles for G protein-regulated adenylyl cyclase isoforms: Insights from knockout and overexpression studies. Neurosignals 2008, 17, 5-22. [CrossRef]

20. Wang, S.-C.; Lin, J.-T.; Chern, Y. Novel Regulation of Adenylyl Cyclases by Direct Protein-Protein Interactions: Insights from Snapin and Ric8a. Neurosignals 2009, 17, 169-180. [CrossRef]

21. Zhang, G.; Liu, Y.; Ruoho, A.E.; Hurley, J.H. Structure of the adenylyl cyclase catalytic core. Nature 1997, 386, 247-253. [CrossRef] 
22. Perreault, M.L.; Hasbi, A.; O'Dowd, B.F.; George, S.R. The Dopamine D1-D2 Receptor Heteromer in Striatal Medium Spiny Neurons: Evidence for a Third Distinct Neuronal Pathway in Basal Ganglia. Front. Neuroanat. 2011, 5, 31. [CrossRef] [PubMed]

23. Tesmer, J.J.G.; Sunahara, R.K.; Gilman, A.G.; Sprang, S.R. Crystal structure of the catalytic domains of adenylyl cyclase in a complex with Gs $\alpha \cdot G T P \gamma$ S. Science 1997, 278, 1907-1916. [CrossRef] [PubMed]

24. Dessauer, C.W.; Tesmer, J.J.; Sprang, S.R.; Gilman, A.G. Identification of a Gi Binding Site on Type V Adenylyl Cyclase. J. Biol. Chem. 1998, 273, 25831-25839. [CrossRef] [PubMed]

25. Frezza, E.; Martin, J.; Lavery, R. A molecular dynamics study of adenylyl cyclase: The impact of ATP and G-protein binding. PLoS ONE 2018, 13, e0196207. [CrossRef]

26. Ho, D.; Yan, L.; Iwatsubo, K.; Vatner, D.E.; Vatner, S.F. Modulation of $\beta$-adrenergic receptor signaling in heart failure and longevity: Targeting adenylyl cyclase type 5. Heart Fail. Rev. 2010, 15, 495-512. [CrossRef]

27. Vatner, S.F.; Park, M.; Yan, L.; Lee, G.J.; Lai, L.P.; Iwatsubo, K.; Ishikawa, Y.; Pessin, J.; Vatner, D.E. Adenylyl cyclase type 5 in cardiac disease, metabolism, and aging. Am. J. Physiol. Circ. Physiol. 2013, 305, H1-H8. [CrossRef]

28. Van Keulen, S.C.; Rothlisberger, U. Exploring the inhibition mechanism of adenylyl cyclase type 5 by n-terminal myristoylated Gai1. PLoS Comput. Biol. 2017, 13, e1005673. [CrossRef]

29. Van Keulen, S.C.; Narzi, D.; Rothlisberger, U. Association of Both Inhibitory and Stimulatory G $\alpha$ Subunits Implies Adenylyl Cyclase 5 Deactivation. Biochemistry 2019, 58, 4317-4324. [CrossRef]

30. Bruce, N.J.; Narzi, D.; Trpevski, D.; Van Keulen, S.C.; Nair, A.G.; Rothlisberger, U.; Wade, R.C.; Carloni, P.; Kotaleski, J.H. Regulation of adenylyl cyclase 5 in striatal neurons confers the ability to detect coincident neuromodulatory signals. PLoS Comput. Biol. 2019, 15, e1007382. [CrossRef]

31. Steegborn, C. Structure, mechanism, and regulation of soluble adenylyl cyclases-Similarities and differences to transmembrane adenylyl cyclases. Biochim. Biophys. Acta 2014, 1842, 2535-2547. [CrossRef]

32. Hurley, J.H. Structure, mechanism, and regulation of mammalian adenylyl cyclase. J. Biol. Chem. 1999, 274, 7599-7602. [CrossRef] [PubMed]

33. Fiser, A.; Sali, A. Modeller: Generation and Refinement of Homology-Based Protein Structure Models. Enzym. Eng. Evol. Gen. Methods 2003, 374, 461-491. [CrossRef]

34. Shen, M.-Y.; Sali, A. Statistical potential for assessment and prediction of protein structures. Protein Sci. 2006, 15, 2507-2524. [CrossRef] [PubMed]

35. Raw, A.S.; Coleman, D.E.; Gilman, A.G.; Sprang, S.R. Structural and Biochemical Characterization of the GTP $\gamma$ S-, GDP·P $\mathrm{i}^{-}$, and GDP-Bound Forms of a GTPase-Deficient Gly ${ }^{42} \rightarrow$ Val Mutant of $\mathrm{G}_{\mathrm{i} \alpha 1}$. Biochemistry 1997, 36, 15660-15669. [CrossRef] [PubMed]

36. Tesmer, J.J.; Berman, D.M.; Gilman, A.G.; Sprang, S.R. Structure of RGS4 Bound to AlF4--Activated Gi $\alpha 1$ : Stabilization of the Transition State for GTP Hydrolysis. Cell 1997, 89, 251-261. [CrossRef]

37. Van Keulen, S.C.; Rothlisberger, U. Effect of N-Terminal Myristoylation on the Active Conformation of Gai1-GTP. Biochemistry 2016, 56, 271-280. [CrossRef]

38. Kozakov, D.; Beglov, D.; Bohnuud, T.; Mottarella, S.E.; Xia, B.; Hall, D.R.; Vajda, S. How good is automated protein docking? Proteins Struct. Funct. Bioinform. 2013, 81, 2159-2166. [CrossRef]

39. Vajda, S.; Yueh, C.; Beglov, D.; Bohnuud, T.; Mottarella, S.E.; Xia, B.; Hall, D.R.; Kozakov, D. New additions to the ClusPro server motivated by CAPRI. Proteins Struct. Funct. Bioinform. 2017, 85, 435-444. [CrossRef]

40. Kozakov, D.; Hall, D.R.; Xia, B.; Porter, K.A.; Padhorny, D.; Yueh, C.; Beglov, D.; Vajda, S. The ClusPro web server for protein-protein docking. Nat. Protoc. 2017, 12, 255-278. [CrossRef]

41. Qi, C.; Sorrentino, S.; Medalia, O.; Korkhov, V. The structure of a membrane adenylyl cyclase bound to an activated stimulatory G protein. Science 2019, 364, 389-394. [CrossRef]

42. Berendsen, H.; Van Der Spoel, D.; Van Drunen, R. GROMACS: A message-passing parallel molecular dynamics implementation. Comput. Phys. Commun. 1995, 91, 43-56. [CrossRef]

43. Lindahl, E.; Hess, B.; Van Der Spoel, D. GROMACS 3.0: A package for molecular simulation and trajectory analysis. Mol. Model. Annu. 2001, 7, 306-317. [CrossRef]

44. Van Der Spoel, D.; Lindahl, E.; Hess, B.; Groenhof, G.; Mark, A.E.; Berendsen, H.J.C. GROMACS: Fast, flexible, and free. J. Comput. Chem. 2005, 26, 1701-1718. [CrossRef]

45. Hess, B.; Kutzner, C.; Van Der Spoel, D.; Lindahl, E. GROMACS 4: Algorithms for Highly Efficient, Load-Balanced, and Scalable Molecular Simulation. J. Chem. Theory Comput. 2008, 4, 435-447. [CrossRef] 
46. Pronk, S.; Páll, S.; Schulz, R.; Larsson, P.; Bjelkmar, P.; Apostolov, R.; Shirts, M.R.; Smith, J.C.; Kasson, P.M.; Van Der Spoel, D.; et al. GROMACS 4.5: A high-throughput and highly parallel open source molecular simulation toolkit. Bioinformatics 2013, 29, 845-854. [CrossRef]

47. Lindorff-Larsen, K.; Piana, S.; Palmo, K.; Maragakis, P.; Klepeis, J.L.; Dror, R.O.; Shaw, D.E. Improved side-chain torsion potentials for the Amber ff99SB protein force field. Proteins Struct. Funct. Bioinform. 2010, 78, 1950-1958. [CrossRef]

48. Wang, J.; Cieplak, P.; Kollman, P.A. How well does a restrained electrostatic potential (RESP) model perform in calculating conformational energies of organic and biological molecules? J. Comput. Chem. 2000, 21, 1049-1074. [CrossRef]

49. Hornak, V.; Abel, R.; Okur, A.; Strockbine, B.; Roitberg, A.; Simmerling, C.L. Comparison of multiple Amber force fields and development of improved protein backbone parameters. Proteins Struct. Funct. Bioinform. 2006, 65, 712-725. [CrossRef]

50. Lindorff-Larsen, K.; Maragakis, P.; Piana, S.; Eastwood, M.P.; Dror, R.O.; Shaw, D.E. Systematic Validation of Protein Force Fields against Experimental Data. PLoS ONE 2012, 7, e32131. [CrossRef]

51. Dolinsky, T.J.; Nielsen, J.E.; McCammon, J.A.; Baker, N.A. PDB2PQR: An automated pipeline for the setup of Poisson-Boltzmann electrostatics calculations. Nucleic Acids Res. 2004, 32, W665-W667. [CrossRef]

52. Jorgensen, W.L.; Chandrasekhar, J.; Madura, J.D.; Impey, R.W.; Klein, M.L. Comparison of simple potential functions for simulating liquid water. J. Chem. Phys. 1983, 79, 926-935. [CrossRef]

53. Dang, L.X. Mechanism and Thermodynamics of Ion Selectivity in Aqueous Solutions of 18-Crown-6 Ether: A Molecular Dynamics Study. J. Am. Chem. Soc. 1995, 117, 6954-6960. [CrossRef]

54. Meagher, K.L.; Redman, L.T.; Carlson, H.A. Development of polyphosphate parameters for use with the AMBER force field. J. Comput. Chem. 2003, 24, 1016-1025. [CrossRef] [PubMed]

55. Allnér, O.; Nilsson, L.; Villa, A. Magnesium Ion-Water Coordination and Exchange in Biomolecular Simulations. J. Chem. Theory Comput. 2012, 8, 1493-1502. [CrossRef]

56. Darden, T.; York, D.; Pedersen, L. Particle mesh Ewald: AnN·log (N) method for Ewald sums in large systems. J. Chem. Phys. 1993, 98, 10089-10092. [CrossRef]

57. Essmann, U.; Perera, L.; Berkowitz, M.L.; Darden, T.; Lee, H.; Pedersen, L.G. A smooth particle mesh Ewald method. J. Chem. Phys. 1995, 103, 8577-8593. [CrossRef]

58. Hess, B.; Bekker, H.; Berendsen, H.J.C.; Fraaije, J.G.E.M. LINCS: A linear constraint solver for molecular simulations. J. Comput. Chem 1997, 18, 1463-1472. [CrossRef]

59. Berendsen, H.J.C.; Van Gunsteren, W.F.; Barnes, A.J. Molecular Liquids-Dynamics and Interactions. In Proceedings of the NATO Advanced Study Institute on Molecular Liquids, Dordretch, The Netherlands, 1 April 1984; pp. 475-500.

60. Harvey, S.C.; Tan, R.K.Z.; Cheatham, T.E., III. The flying ice cube: Velocity rescaling in molecular dynamics leads to violation of energy equipartition. J. Comput. Chem. 1998, 19, 726-740. [CrossRef]

61. Berendsen, H.J.C.; Postma, J.P.M.; Van Gunsteren, W.F.; DiNola, A.; Haak, J.R. Molecular dynamics with coupling to an external bath. J. Chem. Phys. 1984, 81, 3684-3690. [CrossRef]

62. Bussi, G.; Donadio, D.; Parrinello, M. Canonical sampling through velocity rescaling. J. Chem. Phys. 2007, 126, 014101. [CrossRef]

63. Parrinello, M. Polymorphic transitions in single crystals: A new molecular dynamics method. J. Appl. Phys. 1981, 52, 7182. [CrossRef]

64. Daura, X.; Gademann, K.; Jaun, B.; Seebach, D.; Van Gunsteren, W.F.; Mark, A.E. Peptide Folding: When Simulation Meets Experiment. Angew. Chem. Int. Ed. 1999, 38, 236-240. [CrossRef]

65. Laskowski, R.A. SURFNET: A program for visualizing molecular surfaces, cavities, and intermolecular interactions. J. Mol. Graph. 1995, 13, 323-330. [CrossRef]

66. Jones, S.; Thornton, J.M. Principles of protein-protein interactions. Proc. Natl. Acad. Sci. USA 1996, 93, 13-20. [CrossRef]

67. Lee, B.; Richards, F. The interpretation of protein structures: Estimation of static accessibility. J. Mol. Biol. 1971, 55, 379-400. [CrossRef]

68. Hahn, D.K.; Tusell, J.R.; Sprang, S.R.; Chu, X. Catalytic Mechanism of Mammalian Adenylyl Cyclase: A Computational Investigation. Biochemistry 2015, 54, 6252-6262. [CrossRef] 
69. R Development Core Team. R: A Language and Environment for Statistical Computing; R Foundation for Statistical Computing: Vienna, Austria, 2011; ISBN3-900051-07-0. Available online: http://www.R-project.org/ (accessed on 19 August 2011).

70. Wickham, H. ggplot2: Elegant Graphics for Data Analysis; Springer: New York, NY, USA, 2016; ISBN 978-3319-24277-4

71. Pettersen, E.F.; Goddard, T.D.; Huang, C.C.; Couch, G.S.; Greenblatt, D.M.; Meng, E.C.; Ferrin, T.E. UCSF Chimera-A visualization system for exploratory research and analysis. J. Comput. Chem. 2004, 25, 1605-1612. [CrossRef]

72. Frezza, E.; Martin, J.; Lavery, R. A molecular dynamics study of adenylyl cyclase: The impact of ATP and G-protein binding. Zenodo 2018. [CrossRef]

73. Zhu, H.; Domingues, F.S.; Sommer, I.E.C.; Lengauer, T. NOXclass: Prediction of protein-protein interaction types. BMC Bioinform. 2006, 7, 27. [CrossRef]

74. Lensink, M.F.; Velankar, S.; Wodak, S.J. Modeling protein-protein and protein-peptide complexes: CAPRI 6th edition. Proteins: Struct. Funct. Bioinform. 2016, 85, 359-377. [CrossRef]

75. Lambright, D.G.; Noel, J.P.; Hamm, H.E.; Sigler, P.B. Structural determinants for activation of the $\alpha$-subunit of a heterotrimeric G protein. Nature 1994, 369, 621-628. [CrossRef] [PubMed]

(C) 2020 by the authors. Licensee MDPI, Basel, Switzerland. This article is an open access article distributed under the terms and conditions of the Creative Commons Attribution (CC BY) license (http://creativecommons.org/licenses/by/4.0/). 